\title{
Towards Improved Performance and Interoperability in Distributed and Physical Union Catalogues
}

\section{George Macgregor \& Fraser Nicolaides}

\author{
George Macgregor (george.macgregor@strath.ac.uk) is a Researcher at the Centre for Digital Library \\ Research (CDLR), Department of Computer and Information Sciences, University of Strathclyde, \\ Glasgow, UK. \\ Fraser Nicolaides (f.nicolaides@greenwich.ac.uk) is Academic Services Librarian at the University of \\ Greenwich, London, UK and formerly of the M25 Systems Team, London School of Economics \\ Library, London, UK.
}

Keywords: Z39.50, online catalogues, union catalogues, distributed searching, cataloguing, information retrieval, interoperability, SRW

\section{Structured Abstract}

Purpose of this paper: This paper details research undertaken to determine the key differences in the performance of certain centralised (physical) and distributed (virtual) bibliographic catalogue services, and to suggest strategies for improving interoperability and performance in, and between, physical and virtual models.

Design/methodology/approach: Methodically defined searches of a centralised catalogue service and selected distributed catalogues were conducted using the Z39.50 information retrieval protocol, allowing search types to be semantically defined. The methodology also entailed the use of two workshops comprising systems librarians and cataloguers to inform suggested strategies for improving performance and interoperability within both environments.

Findings: Technical interoperability was permitted easily between centralised and distributed models, however the various individual configurations permitted only limited semantic interoperability. Significant prescription in cataloguing and indexing guidelines, greater participation in the Program for Collaborative Cataloging (PCC), consideration of future 'FRBR' migration, and greater disclosure to end users are some of the suggested strategies to improve performance and semantic interoperability.

Practical implications: This paper informs the LIS research community and union catalogue administrators, but also has numerous practical implications for those establishing distributed systems based on Z39.50 and SRW, as well as those establishing centralised systems.

What is original/value of the paper?: The paper moves the discussion of Z39.50 based systems away from anecdotal evidence and provides recommendations based on testing and is intimately informed by the UK cataloguing and systems librarian community. 


\section{Introduction}

Union catalogues are by no means a new phenomenon. As Cannel and Guy (2001) note, the emergence of library cooperatives in the 1970s stimulated the evolution of cooperative cataloguing systems and resource sharing, which in turn began the evolution of what would currently be termed a centralised 'union catalogue'. Perhaps the most obvious example came from the USA where the Ohio College Libraries Center (OCLC) successfully developed automated systems designed to expedite the goals of the cooperative. Such initiatives tended to produce catalogue records for participating libraries either in Computer Output Microfilm (COM) or on catalogue cards to be consolidated with the other catalogue cards. Enthused by developments across the Atlantic, a series of similar initiatives, some more successful than others, were developed in the UK, including the Birmingham Libraries Cooperative Mechanisation Project (BLCMP), South West Academic Libraries Cooperative Automation Project (SWALCAP), and the Scottish Libraries Cooperative Automation Project (SCOLCAP) (Tedd, 1994). Of course, by 1979 the shared cataloguing system used by OCLC had attracted libraries, not just from across the USA, but from across the world, and by 1981 OCLC decided to change their name to the OCLC Online Computer Library Center (Jordan, 2003). Today the OCLC with which we are most familiar has become a leading international library organisation and presides over the largest union catalogue in the world: WorldCat (OCLC, 2004).

As user requirements and expectations have grown in tandem with massive Web development, making these union catalogues accessible to academics and students has long been a keen area of interest for LIS practitioners and researchers around the globe. The main focus for UK activity was the Consortium of University Research Libraries (CURL). CURL recognised early on that their centralised shared cataloguing database (established in 1987) constituted a valuable resource for the academic community and that access to such a resource should be widened (Cousins, 1997). By 1995 CURL had established the CURL OPAC (Copac), providing Web access to the consolidated records of the 26 library members.

Such developments have typically made union catalogues more end-user orientated and since the mid1990s union catalogues have tended to assume one of two manifestations: the centralised (or physical) model and the distributed (or virtual) model. Simply, the former model constitutes a centralised approach whereby bibliographic records contributed from a number of participating institutions are incorporated into a single database. The latter seeks to deliver the same service via a distributed model, most commonly utilising the Z39.50 information retrieval protocol (Z39.50, 2004). Indeed, the increasing pervasiveness of Z39.50 'broadcast searching' has thus allowed participating institutions to remain 'distinct' and to avoid the maintenance costs typically associated with administering a large centralised system (Gatenby, 2002).

As with most technical service models, each has numerous advantages and disadvantages. Some of these have been widely documented in the literature for some time (Cousins, 1999; Nicholson, 2000; Stubley et al, 2001; Gatenby, 2002; Friesen, 2002; Taylor, 2003) or examined (Moen, 2001a; Moen, 
2001b; Moen \& Murray, 2003), whilst others have undergone thorough analysis under the auspices of the UK CC-interop project (Nicolaides, 2003; Gilby \& Sanders, 2003; Gilby et al, 2004; Dunsire \& Macgregor, 2004). Nevertheless, with increasing library Z39.50 compliance the creation of larger heterogeneous distributed union catalogues becomes ever more likely, and issues pertaining to the relative performance of each model have consequently been drawn into sharp focus. The need for improved performance is now also essential to secure the confidence of end users, some of whom believe union catalogue services to be unreliable or irrelevant (Booth \& Hartley, 2004). This focus has sharpened yet further as it becomes clear that those technologies expected to eventually supersede Z39.50 entirely, Web Services Technologies (WST), still harbour various protocol limitations and often suffer from reliability, security and transaction time difficulties (Yu \& Chen, 2003).

Z39.50's recent offshoot initiative, 'Z39.50 International: Next Generation' (ZING), have been fronting several exciting developments, particularly Search/Retrieve Web Service (SRW) and Search/Retrieve URI (SRU) (ZING, 2004). Both SRW and SRU represent an attempt to amalgamate the powerful capabilities of Z39.50 by implementing them in parallel with updated Web-friendly protocols and technologies, such as HTTP (Hypertext Transfer Protocol) with SOAP (Simple Object Access Protocol), a protocol for XML (Extensible Markup Language) messaging, and by utilising WSDL (Web Services Definition Language) to define the Z39.50 messages. Whilst ZING promises greater functionality, and although developers are beginning to incorporate SRW/SRU facilities within standard Z39.50 software (Index Data, 2004), developments remain tentative and some would argue that it will be some time before it becomes as widely accepted as Z39.50. Many libraries have recently invested significant resources to become Z39.50 compliant and it is only now that Z39.50 compliance has truly reached the 'critical mass' to which the UK's Electronic Libraries programme originally aspired in 1998 (Macgregor, 2005).

Still, although Z39.50 has a long history, it is far from outmoded. As Taylor (2003) notes and predicts, Z39.50 may have peculiar problems but it remains capable of adapting to new environments and will experience wider deployment within the LIS sector and beyond for many years. Such predictions are certainly manifest in wider LIS deployment. As in many information rich countries, the UK is experiencing an increasing deployment of Z39.50 applications. While this is most marked in academic and research libraries, it is extending also to Further Education (FE) Colleges, public libraries and lifelong learning institutions. For example, the Scottish Portals for Education, Information and Research project (SPEIR) has spearheaded the wider roll out of Z39.50 across these sectors in order to facilitate the creation of a Scottish Distributed Digital Library (SDDL) (Dunsire \& Macgregor, 2003; Nicholson et al, 2004). The emergence of large scale initiatives in museums provides yet further evidence of Z39.50's deployment potential, and reaffirms the possibilities that wait in creating truly heterogeneous distributed union catalogues (Caplan \& Haas, 2004). Quite simply Z39.50 is, and will remain for the immediate future, the "eminent enabling technology for distributed, parallel access to information sources" (Hammer \& Andresen, 2002). 


\section{Research Purpose \& Objective}

Given this premise, and the growing expectations of user groups, it is essential to improve the performance of both physical and virtual union catalogue models. Moreover, improving performance of each is essential to improving interoperability between both models. Such reasoning has assumed greater relevance via the CC-interop project and, in particular, the work documented by Gilby and Sanders (2003), whereby it is possible to treat an entire virtual union catalogue as a single Z39.50 target (or Z-target) during traditional Z39.50 broadcast searching. This presupposes the future preeminence of ZING technology since SRW/SRU will not provide relief in respect to semantic interoperability and those variations in cataloguing and indexing practices that continue to impair optimal performance of virtual union catalogues. Therefore, the overarching purpose of this paper (and study) is twofold: To identify key differences in the performance of certain centralised (physical) and distributed (virtual) bibliographic catalogue services; To suggest strategies for improving interoperability and performance in, and between, physical and virtual models.

The distinct nature of the research objectives will be reflected in the format of the paper, which will essentially follow two parts. Before discussing the research questions however, it is worthwhile contextualising our study within the remit of the CC-interop project, under the auspices of which much of the said research was undertaken.

\section{Background: The CC-interop project}

In 1998 the UK's Joint Information Systems Committee (JISC) funded the third phase of the Electronic Libraries Programme (eLib), entailing the creation of several virtual union catalogue services (or 'clumps' as they became known). Although the creation of widely used and successful services was an objective, the ultimate aim of the clumps was to "kick start critical mass" in the use of Z39.50 and to generate model technical architectures and agreements to precipitate the development of new clumps in their assorted incarnations, perhaps even nationally (Whitelaw \& Joy, 2001, p.2).

By 2000 four clumps had been created: M25 Link, CAIRNS, RIDING and Music Libraries Online (MLO). All these projects successfully established fully functioning clumps, each with common and unique features. Most were regionally defined and were built upon existing library co-operatives. For our purposes however, the two most significant clumps were M25 Link and CAIRNS:

- M25 Link had six partners drawn from the M25 Consortium of Academic Libraries based in the London area (http://www.m25lib.ac.uk). The eventual distributed catalogue, now comprising 36 institutional Z-servers, forms part of the InforM25 service. It is maintained for the consortium by the M25 Systems Team;

- The Co-operative Academic Information Retrieval Network for Scotland (CAIRNS) (http://cairns.lib.strath.ac.uk/) included members of the Scottish Confederation of University 
and Research Libraries (SCURL) and is now developed and maintained by the Centre for Digital Library Research (CDLR) at the University of Strathclyde. CAIRNS comprises 33 institutional Z-servers, including numerous non-HE Z-servers.

To build on the results and findings of eLib phase three, JISC provided a two-year funding grant to the Copac/Clumps Continuing Technical Cooperation Project (CC-interop: http://ccinterop.cdlr.strath.ac.uk/), which aimed to "bring together, in a virtual modus operandi, distributed catalogues to facilitate richer search and retrieval possibilities for users" (Gilby \& Dunsire, 2004, p.4). Beginning in mid-2002, CC-interop was a collaborative project involving the M25 Systems Team, CDLR, Manchester Information and Associated Services (MIMAS), RIDING, and latterly the Centre for Research in Library and Information Management (CERLIM). The inclusion of the Copac service (http://copac.ac.uk/) at MIMAS - a physical union catalogue established on the consolidated bibliographic records of CURL and searching some 30 million bibliographic records - epitomised the cooperative nature of the project and further emphasised the dialectic nature of the research being undertaken.

The project comprised three work packages, each investigating a plethora of issues, including:

- Inter-linking between very large physical union catalogues (i.e. Copac) and large virtual union catalogues (i.e. InforM25)

- $\quad$ The ability to 'clump the clumps' thus producing a 'hyper-clump'

- Thorough research of collection-level description requirements for such environments

- Improving interoperability in distributed and physical environments

- Investigating user requirements and behaviour for union catalogues

For a greater discussion of the project outcomes and findings refer to Gilby and Dunsire (2004).

\section{Methodology (1)}

Recall our first objective was to identify key differences in the performance of certain centralised (physical) and distributed (virtual) bibliographic catalogue services. Copac was therefore used as the physical union catalogue for study. The distributed services selected for testing were those Consortium of University and Research Libraries (CURL) institutions that were also members of InforM25 (Figure 1). The focus of the performance evaluation was to determine why any given query might elicit a different result set from each of the two types of system. As such, consideration was given to several aspects of the respective systems: from their interpretation of the structured format in which the queries were submitted; to the policies and practices affecting the indexes against which the query was executed. This necessary approach therefore limited the use of quantitative techniques, and instead a methodical qualitative approach was adopted from which broader conclusions could be drawn. 


\section{Searching and Search Types}

Searches of Copac and each of the selected distributed catalogues were conducted using the Z39.50 information retrieval protocol and connections to the relevant Z-servers were made using a Yaz Zclient (Index Data, 2004). This allowed search types to be semantically defined in ways additional to those publicly available through the Copac and InforM25 search interfaces. Searches were constructed using the Bib-1 attribute set, a standard used in Z39.50 to define how search terms are to be treated by the local catalogue (Z39.50, 2003). The search types used for this study included: Author, Author/Title, Key Title (serial title), Subject, Any (keyword). Searches of these types were most inclined, we hypothesised, to elicit a different result set from each system since such search types are subject to greater variation in index scope and content, particularly Author and Subject searches.

No attempt was made to assess the precision of the result sets. This concept is wholly dependent upon the definition of relevance and, as such, was beyond the scope of our research. Instead, in examining the relative performance of Copac and one or more of the distributed systems, we have sought to account for any differences in result set content (in short, why certain records might be present or absent). It is also worth noting that in examining result sets from the centralised and distributed systems, we have been concerned to identify comparable bibliographic records. Any assessment of the presence or quality of any associated holdings and location data (number of copies, enumeration and chronology, etc.) has therefore been omitted.

Although certain significant differences were observed with respect to the capabilities of the examined services, the superiority of either the physical or distributed model is, and will not be, inferred. Rather, the primary concern was to consider the opportunities for effecting greater interoperability between all components, particularly via any potential operational scenarios, such as within a UK National Union Catalogue (UKNUC). Moreover, it was not the purpose of this research to describe all of the potential or current functionality of Copac, or the several distributed Z-servers.

\section{Caveats}

Not all the distributed systems were included in each test. In order of importance, there were three reasons for this:

- As would be expected, all the tests perforce included Copac. However, not all of the distributed systems were enabled to use precisely the sets of Bib-1 attributes supported by Copac.

- In some circumstances, the various institutional implementations of a particular system type performed in a consistent way. Testing more than one implementation was consequently not always necessary. 
- Duplicate searches were not performed when it was felt that the issue or aspect of performance had already been adequately illustrated.

\section{Findings}

The study revealed a variety of differences in system performance between the physical and virtual models. Based on the characteristics of these observations, and to provide greater focus for the results discussion, the authors have deemed these differences to fall into three broad categories: 'Consolidated and Individuated Indexes' (issues pertaining to those indexes in the tested physical and union catalogues respectively), 'Data Currency and Comprehensiveness' (issues pertaining to the currency and comprehensiveness of the records retrieved), and the 'Support and Treatment of Bib-1 Attributes' (the manner in which the tested systems interpreted the search queries and any issues therein).

\section{Consolidated and Individuated Indexes}

As an example of the physical model, the Copac system exploits a feature peculiar to union catalogues in that any bibliographic entity is able to derive index entries from records submitted by several institutions. As would be expected from any centralised system, testing demonstrated that entities have been catalogued to various degrees of comprehensiveness. Bearing in mind that any (consolidated) Copac record may be cumulatively enriched by successive contributing institutions, the potential of the search process to retrieve relevant records proportionately improves. Of course, the corollary dictates that any mis-catalogued entity may generate incorrect index entries, and thus reduce the precision of affected result sets. Although one such instance was encountered during testing, the authors deem this to be a comparatively minor problem given the wider benefits.

\section{- Take in Figures 2 \& 3 -}

By way of example, a right truncated author query for 'greene, $g$ ' was submitted to Copac and to the distributed SAS Z-server. This generated three hits from Copac and one on the SAS Z-server which was duplicated in the Copac result set. The two additional records retrieved from Copac are shown in Figure 2. Both records are present on the SAS catalogue, being found using a similarly structured 'Author' search for 'low, david' and 'gerard, john'. Their retrieval from Copac is a function of the additional indexing of subfield 700\$a, Author Added Entry. In both cases, this contains the term 'Greene, Graham', which matches the requirements of the query. As can be observed from Figure 3, the equivalent records from SAS do not contain this supplemental field. The same occurrence was observed in respect of the testing against Copac and Wellcome, and Copac and the LSE and Imperial respectively, using an Author query without truncation.

The different performance of the two systems may therefore be attributed to the different composition of the records from which the indexes are derived. For Copac, the index entries for each of these items 
have been derived from the relevant records of the multiple contributing institutions. This is indicated in Figure 2 by the multiple instances of the 948 institutional-holdings field. In each case, at least one of these contributions contained the Author Added Entry subfield.

Some variations in the indexing policies and practices of the reviewed institutions and services were also identified. Thus, for any bibliographic record held simultaneously on Copac and the contributing institution's database, index entries may have been derived from differing sets of (sub)fields. This problem is further exacerbated by possible variations in the mapping from the indexes to the Bib-1 Use (access point) attributes. For example, an institution or service may have created several 'Author' indexes, each of which is derived from a differing set of relevant fields and each of which is mapped to a different Use attribute (Author (1003); Name (1002); Personal Name (1); Author - Personal Name (1004); etc. What constitutes any given 'Author' index could therefore vary considerably between databases of essentially the same records.

\section{Data Currency and Comprehensiveness}

Copac maintain an 'update' page (http://copac.ac.uk/about/updated/) to keep users informed as to the currency of the database. Although Copac receives updates from the British Library weekly, updates from other contributing libraries may not be as frequent. As such, the result-sets obtained from the distributed systems were, by and large, found to be more up-to-date than the equivalent sets from Copac. These instances largely concerned a single institution (UCL), which at time of testing had last submitted data to Copac in August 1999. The particular problem encountered in the tests was that records on the institutional database were absent from Copac. (The theoretical corollary is that records pertaining to items withdrawn from stock may still appear on the union database). This issue concerns the relative frequency with which records are updated on local and third-party databases, such as Copac.

One such example was observed when testing the search responses from UCL and Copac. The author query (without truncation) 'capote, truman', returned six records from Copac and eight from UCL. The two additional records from UCL can be seen in Figure 4. ISBN searches on Copac revealed that both items were in fact recorded on the Copac database, but neither had a current holdings statement for UCL (in the 948 field). This discrepancy evidently occurred due to the temporary obsolescence of Copac's UCL data.

\section{- Take in Figure 4 -}

Such discrepancies were also manifest in the 'policy determined' omission of records relating to certain classes of online resource. Indeed, at time of testing, both LSE and Imperial had elected not to submit records to Copac for those bibliographic records describing (and providing links to) electronic resources, such as licensed full-text services or equivalent resources. Conversely, these records were 
accessible to any third-party Z-client through the institutional Z-servers; though, for consistent policy application and service delivery, it is arguable that they should not be.

Whilst it is unnecessary to over-emphasise the importance of what essentially are administrative processes, such discrepancies as outlined above will tend to undermine coherent and consistent results across physical and virtual union catalogues, and could potentially deliver inaccurate results within a hyper-clump environment (particularly one incorporating several third party databases).

\section{Support and Treatment of the Bib-1 Attributes}

As explained previously, the Bib-1 attribute set is designed to enable the definition of all semantic structures relevant to the identification of bibliographic records. Simply, the Bib-1 attribute set provides semantic definition to the search types by deciding their precise nature. For example, a title search for 'ancient american civilizations' (Figure 5) could be interpreted in several ways. Even if it was interpreted by the system as a 'title keyword' search, the issue of truncation still has to be resolved before the search can be undertaken. Such searches are defined by the Bib-1 attribute set in an attempt to resolve these issues pertaining to query interpretation. Figure 6 outlines a basic attribute set for 'title keyword' or 'title exact match' searches, as defined by Use, Relation, Position, Structure, Truncation and Completeness.

\section{- Take in Figure 5 \& 6 -}

To calculate the potential number of attribute combinations would itself be a mathematical challenge. Attribute combinations have consequently been subject to further specification via internationally recognised library profiles such as the Bath Profile (Bath Profile Maintenance Agency, 2004). As an adjunct to the development of commercial and public-domain Z39.50 services, the adoption of Bib-1 has, understandably, been somewhat selective, as has adherence to the related profiles. Thus, in practice, some systems do not support all six attribute types, and, more commonly, most systems support only a selection of the individual attributes and attribute combinations. In many cases, this selectivity has been determined by the limitations of catalogue indexes and local database search routines to which the attributes are mapped. Nevertheless, these tests have revealed several noteworthy aspects of Z-server support and behaviour.

The very scope of the tests was determined by the comparatively limited extent of mutual support for specific attributes and attribute sets. This was patently manifest in the variable support for the 'Position' attributes, First- and Anywhere-in-Field, which perforce can markedly influence retrieval. In some instances, where an attribute (or attribute combination) was not supported, it was replaced with an alternative attribute (or combination) by the Z-server. Such default Z-server behaviour was exemplified with the treatment of 'Truncation' by those distributed services using Unicorn systems 
(Imperial and LSE) and can impact significantly on the consistency of the result sets obtained from differently implemented databases of the same records.

A distinctive variation on this concerns Copac's ability to interpret the query term (rather than, or in addition to, the attribute set). Testing detailed an 'Author' search that, because the term was in a normalised format was, by definition, submitted to a 'Quick Author' index. This operation effectively negated one of the specified attributes, 'No Truncation', a function that is actually supported by Copac. Whilst these various default modifications are usually intended to maximise the efficiency of the search and to optimise system performance in a one-to-one (Z-client to single Z-server) relationship, it may not be entirely appropriate in operational environments where a Z-client wishes to affect some measure of semantic consistency between multiple Z-servers.

One final notable behaviour of the tested services related to the processing of the 'Structure' attribute, 'Phrase'. As we discovered, Copac and certain institutional Z-servers responded differently to words within queries that were defined as stopwords or those that could be interpreted as Boolean operators.

In short, the relatively few systems that we examined have been shown to support disparate varieties of search type (semantically defined using the Bib-1 attribute set) and the consequent requirement for some measure of semantic interoperability is clearly evident.

\section{Implications}

Although the small scale of the study does not permit us to be absolutely authoritative in determining the relative importance of each of the above issues, it is reasonable to assume that the least important concerned the time-lag with which records were updated on the centralised database. Remedial action, if indeed it were deemed to be necessary, would essentially require an organisational rather than a technical solution. The two other factors, to which equal importance should be ascribed, concern variations in, firstly, cataloguing and indexing practices and, secondly, support for Bib-1 attributes and attribute sets.

A general observation made by Heiler (1995, p.271) continues to ring true: "Semantic agreements are often lacking when old data or procedures are used for new purposes not anticipated by their original developers". Variations in cataloguing and indexing policies and practices have long been recognised within the Z39.50 community as an impairment to semantic interoperability (Lynch, 1997; Moen, 2001b; Nicholson et al, 2001; Friesen, 2002). This affects all search types, but is perhaps most pronounced for 'Subject' and 'Keyword' type searches. Such variations are, of course, the product of historical and local requirements and contingencies, the legitimacy of which should not be challenged. Nevertheless, such issues are all pervasive and, as Simeoni (2004) notes, are beginning to blight the performance of Federated Digital Libraries (FDLs) founded upon OAI-PMH also. 
Nicholson and Shiri (2003) note that semantic interoperability constitutes the largest obstacle to providing coherent distributed digital libraries, and although McCulloch (2004) and McCulloch et al (2005) note some of the exciting 'terminology mapping' developments and initiatives underway to provide a technical solution to these problems, it is not unreasonable to assume that it will be many years before such solutions are capable of being readily deployed within distributed digital library architectures. As McCulloch (2004) observes, even before such solutions can take root, information providers need to champion and implement international standards where multiple terminologies are in use. Operational difficulties might arise with the possibly historical use of multiple schemes, the use of ad hoc institutionally specific schemes, the irregular application of schemes, and so forth.

Of course, the scope of the Bib-1 attribute set has allowed for multiple disparate implementations to be made, as demonstrated by the variations in those of Copac and each of the distributed services under analysis. Within many profiles, semantic interoperability is addressed through the definition of a core suite of search types (constructed using specified sets of attributes). For the current and possible future application scenarios in which Copac and the distributed systems might operate, the most relevant profile would be the Bath Profile since certain commercial vendors supplying Z-server modules to higher-educational and other institutions are committed to adoption of the Profile (Nicolaides, 2003b).

The technical challenges, however, should not be underestimated. In order to support the required attribute sets, it may be necessary for a library to engage in technically demanding and financially onerous tasks, such as re-indexing their catalogue. Essentially, the technical interoperability of Copac with other distributed systems was never in doubt. Rather, what testing documented was that the various individual configurations permit only limited semantic interoperability. Thus, any supranational system (or 'hyper-clump') that seeks to integrate or otherwise utilise such component services must address the above noted issues.

\section{Strategies for Improving Interoperability and Performance}

Given some of these short to medium term challenges, how best can the LIS community improve interoperability and performance of, and between, physical and virtual union catalogues? The most obvious strategy is to initiate some form of coordination of cataloguing and indexing practices. Such concerted initiatives have hitherto been few and far between. Indeed, the only visible attempt in the literature to arrest interoperability problems caused by variations in cataloguing and indexing practices was undertaken by CAIRNS (CAIRNS Cataloguing and Indexing Working Group, 2000). CAIRNS appended to these guidelines a variety of suggested short and long term strategies for alleviating interoperability problems, some of which met with at least nominal success (Nicholson et al, 2001).

Yet, a more general lack of activity is unsurprising since semantic interoperability is inextricably tied to 'communities of practice' (Friesen, 2002). Moen (2001b) clarifies this supposition by defining Networked Information Discovery and Retrieval (NIDR) (of which virtual union catalogues constitute 
one such incarnation) as falling into one of three communities: Focal, Extended and Extra. Moen's definition therefore dictates that the further a virtual union catalogue moves away from a 'Focal' community (typified by minimal interoperability issues and a large degree of homogeneity), the greater the challenges and cost are to achieving true interoperability. By acknowledging the work of Gilby and Sanders (2003), we soon recognise that the potential for creating supra-national distributed catalogues and hyper-clumps will inevitably dictate that member libraries will be party to an 'Extra' community where there exist numerous factors affecting interoperability. Such an assertion doubtless requires the definition of suitable strategies for improving interoperability and performance in, and between, physical and virtual, particularly with respect to coordinating cataloguing and indexing practices to maximise interoperability.

\section{Methodology (2)}

To ascertain which strategies and mechanisms would prove most effective in providing some degree of homogeneity in UK cataloguing and indexing practices, a qualitative approach was adopted whereby the opinions and views of the UK cataloguing and systems fraternity were canvassed. The objectives of this approach were threefold:

- To identify or suggest strategies capable of addressing or alleviating variant cataloguing and indexing practices in the UK.

- To ensure such strategies or proposed recommendations were intimately informed by the UK cataloguing and systems fraternity, thus ensuring the legitimacy and authenticity of championing such strategies in the literature and beyond.

- To ascertain whether a UK wide initiative, based primarily on the CAIRNS experience, could be adopted and whether the UK cataloguing and systems fraternity would be receptive to such an initiative were it to be rolled out at a strategic level.

Two one-day workshops were organised in London (A) and Glasgow (B) respectively. Invitations for workshop A and B were issued on relevant UK email lists. A revised and more generic version of the CAIRNS cataloguing guidelines was then distributed in advance of the workshop and participants were encouraged to review these guidelines in preparation for the event. Participants were also encouraged to bring along examples of policy and practice from their local institutions, and issues they had encountered in using union catalogues, to support, contradict, and otherwise inform the workshops.

Both workshops consisted of a number of short presentations in the morning to outline and to refresh participants of the 'issues', followed by a facilitated and semi-structured group discussion in the afternoon. With permission of the participants, these discussions were tape recorded and, together with notes taken during the session, were amalgamated to produce a report summary of the discussions. 
These reports were then distributed to the participants for amendment, comment and correction, and then consolidated to reflect views expressed at both workshops.

Both events were well attended and attracted representation from many Higher Education and large research libraries, as well as representation from Further Education colleges. In total, 52 attended the workshops.

\section{Findings: Strategies and Recommendations}

A clear consensus emerged at both workshops A and B that the UK cataloguing community requires, and would welcome, the creation of guidelines that were more prescriptive than the current CAIRNS guidelines. Such prescription would assist local cataloguers in actively improving interoperability, whilst simultaneously placing a degree of leverage in the hands of cataloguers and systems administrators to encourage acknowledgement by senior library management of the consequences local policies can have on global interoperability. Nevertheless participants agreed that the continuing globalisation of cataloguing, and the future potential for hyper-clump creation, dictated that it would be more constructive to produce a set of recommendations for a wider, more active and co-ordinated approach to improving interoperability. More specific findings, strategies or recommendations from workshop A and B participants fell into four categories: collaboration within distributed or physical union catalogues; standards; strategic developments; end users.

\section{Collaboration within distributed or physical union catalogues}

Participants at the workshops were unanimous in their recommendation that consortia of libraries contributing to union catalogues should, in the absence of any immediate strategic guidance, develop their own prescriptive guidelines covering catalogue record scope and content, whilst accounting for both local and 'global' needs. Such guidelines might include a minimum input standard for the level of cataloguing and the content of entry points or headings. As argued earlier, it is no longer sufficient for such guidelines to be developed for one mode or level of aggregation. Any one library may belong to more than one union catalogue, requiring local needs to be matched against more than one set of global needs.

By way of example, the National Library of Scotland contributes to the CAIRNS distributed union catalogue, the Copac physical union catalogue, and the British National Bibliography. Any union catalogue may in turn be treated as a single component catalogue of a larger distributed union catalogue; so that what constitutes global in one environment constitutes local in another. Similarly, Strathclyde University Library is a member of CAIRNS, but CAIRNS itself may become a member of a hyper-clump such as a distributed UKNUC. Once again, CAIRNS would be global in the first environment, but local in the second. By continuing this theme yet further, an UKNUC could feasibly become a local component in a distributed union catalogue for the Anglophone world, and so forth. It 
therefore becomes clear that guidelines for improving interoperability need to be developed at national and international levels and suitable mechanisms for doing so should be identified or even created. One such existing mechanism identified by workshop participants for the UK was the Full Disclosure initiative hosted at the British Library (British Library Board, 2004).

Greater participation by consortia in international activities, such as the Program for Collaborative Cataloging (PCC, 2004), should be encouraged. This would reconcile clashes between local and global name and subject headings, and ensure future interoperability with international distributed union catalogues. In addition, consortia should consider developing a shared cataloguing service for digital resources, involving the creation of only one catalogue record to be used, or copied, by all member libraries. Rules for cataloguing digital resources tend to offer more choice, and therefore greater opportunity for variations and increased interoperability difficulties. As workshop participants recognised, there is much less need, if any, for local data in the catalogue record for a resource that is not circulated or shelved.

The role of communication was also identified by cataloguers and systems librarians as particularly important to ensuring that local reviews pertaining to cataloguing and indexing practice resonate with the wider globalisation of bibliographic records. Though email communication was deemed useful, participants agreed that catalogue consortia should develop mechanisms to ensure regular opportunities to discuss issues and review policy or practices. Indeed, participants were unanimous in their concern that providing proper professional advice to colleagues would be unforthcoming if they were unable to discuss views, concerns, and experiences with fellow professionals, or inform themselves of cataloguing developments occurring within their immediate locale. Such concerns appear to be increasing as pressures to reduce costs and develop new services increase and become ubiquitous within LIS circles.

\section{Standards}

Whilst greater acknowledgement of the Bath Profile was deemed necessary amongst libraries, Z39.50 implementers, and library system vendors, it was recognised that further development work on the Bath Profile should encompass recommendations for the scope and content of specified indexes. The Bath Profile offers greater prescription, yet there still remains a plethora of local choices to be made during Z39.50 installation and implementation. Such choices are not informed by global interoperability requirements and constitute further obstacles to improving interoperability. For example, the Title index could be scoped to cover alternate titles, uniform titles, group and part titles, and related titles. Further still, a normalisation rule could be applied to all scoped titles. This, for example, might entail the removal of leading articles, such as 'The' and 'An'. Such improvements in the Bath Profile would give cataloguing consortia, system vendors, and Z39.50 service developers a sound basis for establishing standard index mappings from metadata formats such as MARC21. In any case, participants suggested that consortia using Z39.50 should consider producing guidelines on required 
conformance with the Bath Profile, specifying conformance areas and specific indexes and searches. This would be more prescriptive than the Profile itself, and by reducing choice would arguably improve interoperability.

In addition, standard rules for index content normalisation could be specified (and adopted) at as wide a level as possible. Such rules would obviously cover punctuation in names, titles and subjects, the inversion of personal names, and the treatment of leading articles in titles. Standard rules would allow system vendors and service developers to ensure more uniformity in Z-indexes. The adaptation of existing rules, such as those used by the Name Authority Control (NACO), was deemed by workshop participants to be feasible and wholly desirable.

\section{Strategic developments}

The most significant strategic development likely to impinge on future library system design is the gradual shift towards IFLA's Functional Requirements for Bibliographic Records (FRBR) model (IFLA, 1998). Indeed, as Tillett (2004, p.7) notes, "vendors and bibliographic utilities like VTLS, OCLC, and RLG have already embraced the FRBR conceptual model in designing their future systems". The FRBR approach is based on an entity-relationship model as a generalised view of the global bibliographic universe. Such a model offers a new perspective on the composition and relationships of bibliographic and authority records, as well as greater precision in the vocabulary used to describe information entities. Whilst the adoption of FRBR has been slower in the US, enthusiastic application in Europe and Australia has compelled the LIS fraternity to begin coordinated planning to ensure a smooth transition.

Consequently, workshop participants noted that consortia and individual libraries should monitor the implementation of FRBR to plan for large-scale machine processing of catalogue data to improve interoperability. Upgrading a cataloguing system to the FRBR model requires disaggregation of existing catalogue record components and reaggregation into a significantly different structure (Delsey, 2004). In particular, the true benefits to be derived from the FRBR model are obtained when the catalogues are used in a global environment. This dictates that the effectiveness of the FRBR model depends on precision in name and title indexes, thus facilitating a degree of automated conversion whereby local records are matched and upgraded against fuller, more authoritative global files (Tillett, 2004). Of course the costs of implementing the FRBR model within a local catalogue are likely to be significant and it was recognised that a better return on investment would be secured if a 'global context' was applied to all operations involving library catalogues where possible, rather than simply upgrading to FRBR because it is what their library system vendor is offering. More significantly, by applying a global context to all operations, interim and future interoperability would be improved and the future FRBR-isation of catalogue data optimised and rendered more manageable.

\section{End users}


An intriguing outcome of the workshops was the suggestion that disclosure of local practices could affect interoperability for end-users by influencing their search behaviour. Such information might be embedded within the catalogue interface, or offered via help, orientation, or training screens. Opinion was divided as to how frank such information should be, particularly if it emphasised potentially negative issues such as incomplete catalogues or poor-quality records. In point of fact, many questioned whether end-users would be interested in, or use, this kind of support. Other participants suggested that service administrators may incur the displeasure of more experienced searchers (academics, research staff, etc.) if they were not informed of those factors that could affect their entire search strategy. Nevertheless, participants were in agreement that consortia should consider agreeing a standard set of information about each catalogue which should be disclosed as part of the union catalogue service, allowing additional information to be disclosed on the local catalogue interface at the discretion of the library.

\section{Conclusions and Wider Relevance}

Whilst the various observations and conclusions drawn in this paper are derived from our analysis of established services within the UK, most if not all of the issues are likely to have an international resonance. The primary purpose of this paper has been to identify key differences in the performance of certain physical and virtual bibliographic catalogue services and to provide illustrative examples, as well as to suggest strategies for improving interoperability and performance in physical and virtual systems. Such assessments have to be undertaken since the future potential for creating ever larger heterogeneous Z-based union catalogues increases in parallel with growing library Z-compliance, thus drawing performance issues into an ever sharper focus.

Miller (2000) identifies several types of interoperability, including technical, semantic, political/human and international interoperability. As a Z-enabled service, the technical interoperability of Copac with other distributed systems was never in doubt. Indeed, as Miller notes, technical interoperability "is the most straightforward aspect of maintaining interoperability, as there are often clear 'right' and 'wrong' answers to be found" (Ibid.). However, what the crux of this paper has sought to illustrate is that the various individual configurations permit only limited semantic interoperability, as evidenced by those issues relating to consolidated indexes, data currency and the support or treatment of Bib-1 Attributes. Perhaps more importantly, these considerations on semantic interoperability will apply equally to SRW which, although lowering the barriers to future Z39.50-style implementations, will suffer from suboptimal performance as a consequence of poor semantic interoperability. Such sub-optimal performance has the potential to be more pronounced in coming years with the increasing prevalence of FRBR, where the requirement for semantic specificity will be essential in order to expedite the coherent and meaningful distributed services that users have come to expect. 
To meet these expectations it is imperative that significant prescription be introduced into any cataloguing and indexing guidelines adopted by library consortia (or a union catalogue service) in order to thwart those variations in cataloguing and indexing practices that are currently compromising services. Such prescription is not only essential to improve interoperability and performance, but is necessary to secure the confidence of end users, some of whom already harbour little confidence in union catalogue services (Booth \& Hartley, 2004). More generally however, greater strategic guidance is required from international LIS bodies to plan for future supra-national catalogues. It is therefore not unfeasible to suggest that prescriptive guidelines for the Anglophone be developed via a partnership of national libraries (Library of Congress, British Library, National Library of Australia, etc.), in tandem with further Bath Profile development as outlined previously. Whilst interoperability at an international level should be aspired to, such a partnership would function as a catalyst for wider international interoperability initiatives and if nothing else, would unquestionably constitute a lesson in 'political/human interoperability'. Nevertheless, greater participation in the PCC, consideration of future FRBR migration, and greater disclosure to end users are all activities in which individual libraries and library consortia can actively influence and improve interoperability. In short, libraries need to think globally before acting locally.

\section{Acknowledgements}

Gratitude is extended to Dr. Shirley Cousins and Ashley Sanders for providing access to Copac records in MARC format and assisting greatly in the interpretation of search results.

Appreciation is also extended to those cataloguers and systems librarians that participated in the cataloguing / interoperability workshops. 


\begin{tabular}{|l|l|l|}
\hline $\begin{array}{l}\text { Distributed Services (Distributed Z- } \\
\text { servers) }\end{array}$ & Abbreviation & Library System \\
\hline $\begin{array}{l}\text { Imperial College of Science, Technology } \\
\text { \& Medicine }\end{array}$ & Imperial & Unicorn \\
\hline London School of Economics & LSE & Unicorn \\
\hline School of Advanced Study & SAS & Innopac \\
\hline University College London & UCL & Aleph \\
\hline University of London Library & ULL & Innopac \\
\hline $\begin{array}{l}\text { Wellcome Library for the History \& } \\
\text { Understanding of Medicine }\end{array}$ & Wellcome & Innopac \\
\hline
\end{tabular}

Figure 1: Table detailing those distributed systems used for the experiment that are members of both InforM25 and Copac.

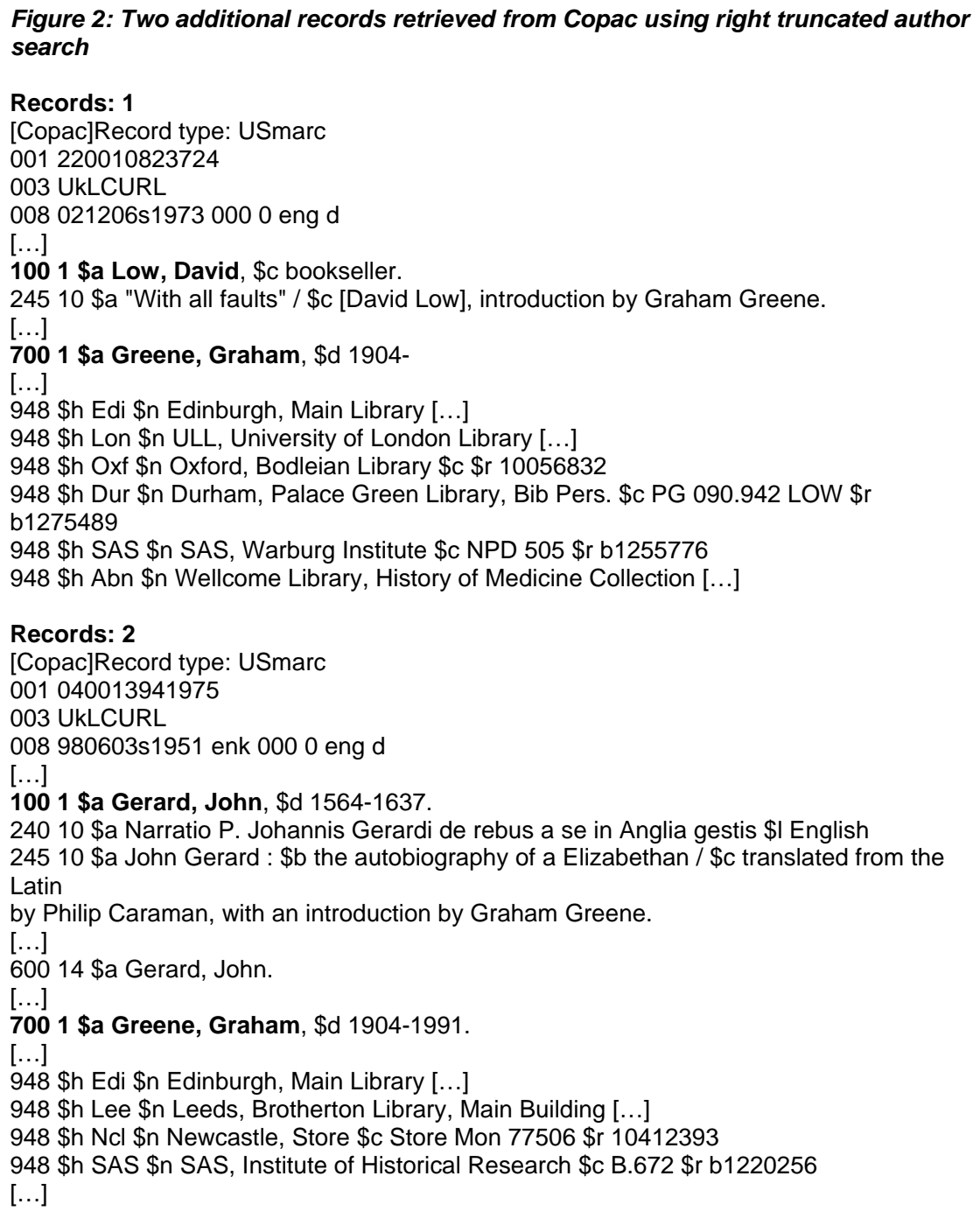




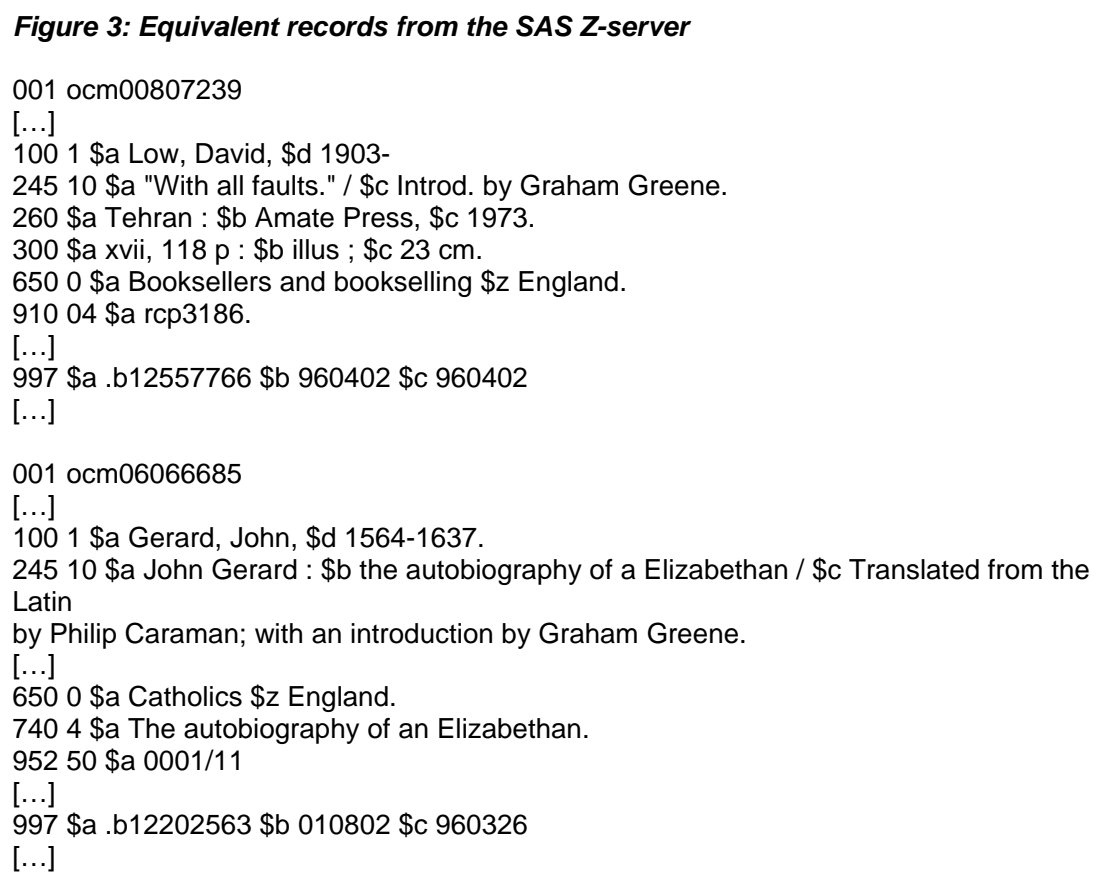

Figure 4: The two additional records from UCL, not found in Copac

\section{Records: 1}

[UCL01]Record type: USmarc

$[\ldots]$

$020 \$ a 0241017815$

1001 \$a Capote, Truman, \$d 1924-1984

$24514 \$$ a The thanksgiving visitor

260 \$c 1967

$[\ldots]$

Records: [2]

[UCL01]Record type: USmarc

001 AC000022099

$[\ldots]$

020 \$a 0878052747 \$c (alk. paper)

020 \$a 0878052755 \$c (pbk. : alk. paper)

[...]

1001 \$a Capote, Truman, \$d 1924-1984

24510 \$a Truman Capote: \$b conversations / \$c edited by M. Thomas Inge.

260 \$a Jackson, Miss. ; \$a London: \$b University Press of Mississippi, \$c c1987.

$[\ldots]$

60010 \$a Capote, Truman, \$d 1924-1984 \$v Interviews

$[\ldots]$ 


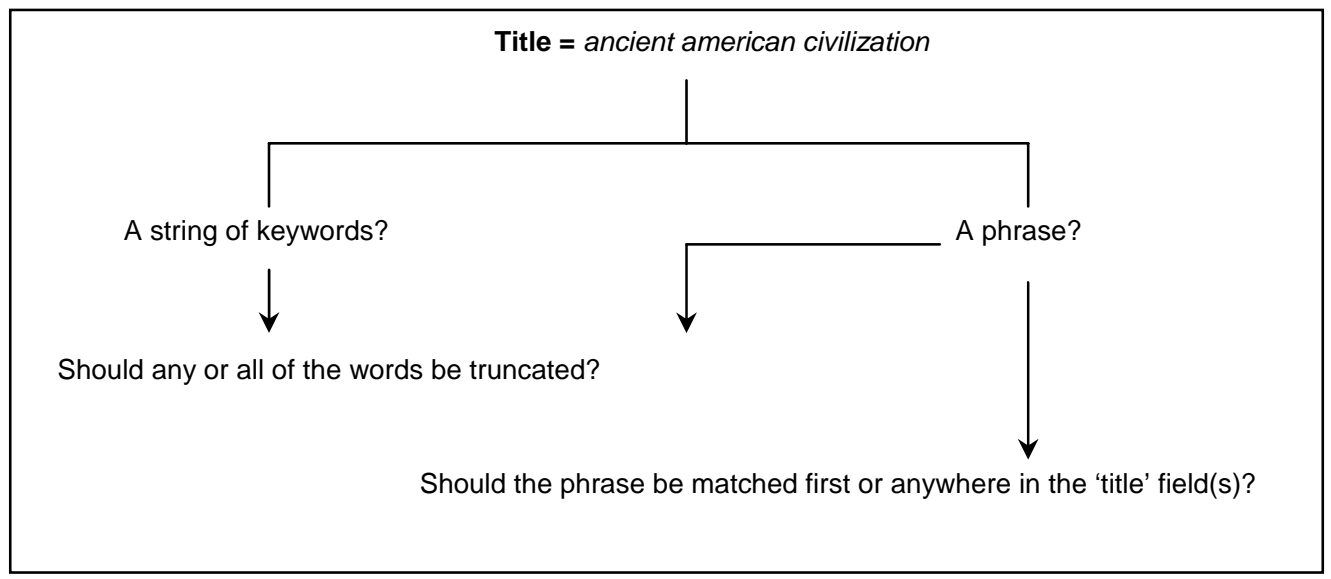

Figure 5: Process of defining the semantic nature of the search query.

\begin{tabular}{|l|l|l|l|l|}
\hline \multirow{2}{*}{ Attribute type } & \multicolumn{2}{|l|}{ Title search - keyword } & \multicolumn{2}{l|}{ Title search - exact match } \\
\cline { 2 - 6 } & Value & Attribute & Value & Attribute \\
\hline Use (1) & 4 & Title & 4 & Title \\
\hline Relation (2) & 3 & Equal & 3 & Equal \\
\hline Position (3) & 3 & Any-position-in-field & 1 & First-in-field \\
\hline Structure (4) & 2 & Word & 1 & Phrase \\
\hline Truncation (5) & 100 & Do not truncate & 100 & Do not truncate \\
\hline Completeness (6) & 1 & Incomplete subfield & 3 & Complete field \\
\hline
\end{tabular}

Figure 6: Basic Bib-1 attribute set for Z39.50 title search. 


\section{References}

Bath Profile Maintenance Agency. (2004), The Bath Profile: An International Z39.50 Specification for Library Applications and Resource Discovery - Release 2.0, Library and Archives of Canada, Ottawa. Available: http://www.collectionscanada.ca/bath/tp-bath2-e.htm (Accessed 12 January 2005)

Booth, H. \& Hartley, R, J. (2004), User behaviour in the Searching of Union Catalogues: An Investigation for Work Package C of CC-interop, Centre for Digital Library Research, Glasgow. Available: http://ccinterop.cdlr.strath.ac.uk/documents/finalreportWPC.pdf (Accessed 12 January 2005)

British Library Board. (2004), What is Full Disclosure? British Library, London. Available: http://www.bl.uk/about/cooperation/fdhome.html (Accessed 12 January 2005)

CAIRNS Cataloguing and Indexing Working Group. (2000), CAIRNS Project Recommendations for a Cataloguing and Indexing Strategy for Scottsih Libraries, University of Glasgow, Glasgow. Available: http://cairns.lib.gla.ac.uk/docs/CAIRNSCatStrat.pdf (Accessed 12 January 2005)

Cannell, S. \& Guy, F. (2001), Cross-Sectoral Collaboration in the Choice and Implementation of a Library Management System: the Experience of the University of Edinburgh and the National Library of Scotland, Program: electronic library and information systems, Vol.35 No.2, pp.135-156.

Caplan, P. \& Haas, S. (2004), Metadata Rematrixed: Merging Museum and Library Boundaries, Library Hi-Tech, Vol.22 No.2, pp.263-269.

Cousins, S, A. (1997), COPAC: the new national OPAC service based on the CURL database, Program, Vol.31 No.1, pp.1-21.

Cousins, S. (1999), Virtual OPACs Versus Union Database: Two Models of Union Catalogue Provision, The Electronic Library, Vol.17 No.2, pp.97-103.

Delsey, T. (2004), Functional Requirements for Bibliographic Records - User Tasks and Cataloguing Data: Part 2, Catalogue and Index, No.151. pp.1-4.

Dovey, M. (2000), So You Want to Build a Union Catalogue?, Ariadne, No. 23. Available: http://www.ariadne.ac.uk/issue23/dovey/intro.html (Accessed 12 January 2005)

Dunsire, G. \& Macgregor, G. (2003), Clumps and Collection Description in the Information Environment in the UK, with Particular Reference to Scotland, Program: electronic library and information systems, Vol.37 No.4, pp.218-225.

Dunsire, G. \& Macgregor, G. (2004), Improving Interoperability in Distributed and Physical Union Catalogues through Co-ordination of Cataloguing and Indexing Policies, Centre for Digital Library Research, Glasgow. Available: http://ccinterop.cdlr.strath.ac.uk/documents/CCICatInterop.pdf (Accessed 12 January 2005)

Friesen, N. (2002), Semantic Interoperability and Communities of Practice, Canadian Core Learning Resource Metadata Applications Profile, St. Edmonton, Canada. Available:

http://www.cancore.ca/documents/semantic.html (Accessed 12 January 2005)

Gatenby, J. (2002), Aiming at Quality and Coverage Combined: Blending Physical and Virtual Union Catalogues, Online Information Review, Vol.26 No.5, pp.326-334.

Gilby, J. \& Dunsire, G. (2004), COPAC/Clumps Continuing Cooperation Project (CC-interop): Final Report, Centre for Digital Library Research, Glasgow. Available: http://ccinterop.cdlr.strath.ac.uk/documents/CCiFinalReportVersion1.pdf (Accessed 12 January 2005)

Gilby, J. \& Sanders, A. (2003), Transforming a Clump into a Z-target: A Feasibility Study, Centre for Digital Library Research, Glasgow. Available: http://ccinterop.cdlr.strath.ac.uk/documents/Clump_ztarget_issue1_0.pdf (Accessed 12 January 2005) 
Gilby, J. , Sanders, A. \& Cousins, S. (2004), Bibliographic Union Catalogue Results and Display Issues, Centre for Digital Library Research, Glasgow. Available:

http://ccinterop.cdlr.strath.ac.uk/documents/WPALastReportIssue1.pdf (Accessed 12 January 2005)

Hammer, S. \& Andresen, L. (2002), Issues in Z39.50 Parallel Searching, Denmark's Electronic Research Library, Copenhagen. Available: http://www.deflink.dk/upload/doc filer/doc_alle/895_Issues\%20in\%20Z39_50\%20Parallel\%20Searchi ng.htm (Accessed 12 January 2005)

Heiler, S. (1995), Semantic Interoperability, ACM Computing Surveys, Vol.27 No.2, pp.271-273.

IFLA. (1998), Functional Requirements of Bibliographic Records: Final Report, K.G. Saur, Munchen. Available: http://www.ifla.org/VII/s13/frbr/frbr.pdf (Accessed 12 January 2005)

Index Data. (2004), Yaz, Index Data, Denmark. Available: http://www.indexdata.dk/yaz/ (Accessed 12 January 2005)

Jordan, J. (2003), OCLC and the Emerging Worldwide Library Cooperative, Library Management, Vol.24 No.3, pp.107-115.

Lynch, C, A. (1997). The Z39.50 Information Retrieval Standard - Part 1: A Strategic View of its Past, Present and Future, D-Lib Magazine, April 1997. Available:

http://www.dlib.org/dlib/april97/04lynch.html (Accessed 12 January 2005)

Macgregor, G. (2005), Z39.50 Broadcast Searching and Z-Server Response Times: perspectives from CC-interop, Online Information Review, Vol.29 No.1. In Press.

McCulloch, E. (2004), Multiple Terminologies: An Obstacle to Information Retrieval, Library Review, Vol.53 No.6, pp.297-300.

McCulloch, E., Shiri, A. \& Nicholson, D. (2005), Challenges and Issues in Terminology Mapping: A Digital Library Perspective, Electronic Library. In Press.

Miller, P. (2000), Interoperability: What is it and why should I want it? Ariadne, 24. Available: http://www.ariadne.ac.uk/issue24/interoperability/ (Accessed 12 January 2005)

Moen, W, E. (2001a). Improving Z39.50 Interoperability: Z39.50 Profiles and Testbeds for Library Applications, In: Proceedings of the 67th International Federation of Library Associations Council and General Conference, Universal Dataflow and Telecommunications Workshop, Boston, MA, USA August 16-25 2001, IFLA, The Hague. Available: http://www.ifla.org/IV/ifla67/papers/050203e.pdf (Accessed 12 January 2005)

Moen, W, E. (2001b), Mapping the Interoperability Landscape for networked Information Retreival, In: Proceedings of First ACM/IEEE-CS Joint Conference on Digital Libraries, Roanoke, VA, USA, June 24-28 2001, University of North Texas, Denton. Available: http://www.unt.edu/wmoen/publications/MapInteropJCDLFinal.pdf (Accessed 12 January 2005)

Moen, W, E. \& Murray, K, R. (2003), Z39.50 Server Implementation Issues and Recommendations, Texas Ceter for Digital Knowledge, Denton. Available: http://www.unt.edu/zlot/Deliverables/WA4_del_z-server_v2_krm_23jun2003.doc (Accesssed 12 January 2005)

Nicholson, D. (2000), Clumping Towards a UK National Catalogue? Ariadne, No.22. Available: http://www.ariadne.ac.uk/issue22/distributed/distukcat.html (Accessed 12 January 2005)

Nicholson, D., Denham, M., Dunsire, G. \& Gillis, H. (2001), CAIRNS Final Report: An Embryonic Cross-sectoral, Cross-domain National Networked Information Service for Scotland?, Glasgow University, Glasgow. Available: http://cairns.lib.gla.ac.uk/cairnsfinal.pdf (Accessed 12 January 2005) 
Nicholson, D. \& Shiri, A. (2003), Interoperability in Subject Searching and Browsing, OCLC Systems \& Services, Vol.19 No.2, pp.58-61.

Nicholson, D., Dunsire, G., Dawson, A., Macgregor, G., Shiri, A., Joseph, A., Williamson, A. \& Jones, E. (2004), Elements and Future Development Requirements of a Common Information Environment for Scotland: Final Report to the Scottish Library and Information Council (SLIC) on the SPEIR Project, Centre for Digital Library Research, Glasgow. Available: http://speir.cdlr.strath.ac.uk/documents/SPEIRPublishedVersions/0SPEIRFINALReport261004.pdf (Accessed 12 January 2005)

Nicolaides, F. (2003a). A Comparative Study of the Performance of COPAC and Selected Independent Z39.50 Servers, Centre for Digital Library Research, Glasgow. Available: http://ccinterop.cdlr.strath.ac.uk/documents/WPA_server_tests_issue1.pdf (Accessed 12 January 2005)

Nicolaides, F. (2003b), The Bath Profile Four Years On: What's being done in the UK? Ariadne, No.36. Available: http://www.ariadne.ac.uk/issue36/bath-profile-rpt/intro.html (Accessed 12 January 2005)

OCLC. (2004), WorldCat at a Glance, OCLC, Ohio. Available: http://www.oclc.org/worldcat/about/default.htm (Accessed 12 January 2005)

PCC. (2004), available: http://www.loc.gov/catdir/pcc/ (Accessed 12 January 2005)

Simeoni, F. (2004), Servicing the Federation: The Case for Metadata Harvesting, In: Heery, R. \& Lyon, L. (ed), Proceedings of the $8^{\text {th }}$ European Conference on Digital Libraries: Research and Advanced Technology for Digital Libraries, Sept. 12-17 2004, Springer, Berlin.

Stubley, P., Bull, R. \& Kidd, T. (2001). Feasibility Study for a National Union Catalogue - Final Report, University of Sheffield, Sheffield. Available: http://www.uknuc.shef.ac.uk/NUCrep.pdf (Accessed 12 January 2005)

Taylor, S. (2003), A Quick Guide to Z39.50, Interlending \& Document Supply, Vol. 31 No. 1, pp.2530.

Tedd, L, A. (1994), OPACs Through the Ages, Library Review, Vol.43 No.4, pp.27-37.

Tillett, B. (2004), What is FRBR? A Conceptual Model for the Bibliographic Universe, Library of Congress Cataloging Distribution Service, Washington DC. Available: http://www.loc.gov/cds/downloads/FRBR.PDF (Accessed 12 January 2005)

TZIG. (2003), Z Texas Profile: A Z39.50 Specification for Library Systems Applications in Texas Release 3.0, Texas State Libraries and Archives Commission, Austin. Available: http://www.tsl.state.tx.us/ld/projects/z3950/tzigprofilerelease30.html (Accessed 12 January 2005)

Whitelaw, A. \& Joy, G. (2001), Summative Evaluation of Phase 3 of the eLib Initiative: Final Report, UKOLN, Bath. Available: http://www.ukoln.ac.uk/services/elib/papers/other/summativephase-3/elib-eval-main.pdf (Accessed 12 January 2005)

Yu, Shien-Chiang \& Chen, Ruey-Shun. (2003). Web Services: XML-based System Integrated Techniques, Electronic Library, Vol. 21, No. 4, pp.358-366.

ZING. (2004), ZING - Z39.50 International: Next Generation, Library of Congress, Washington DC. Available: http://www.loc.gov/z3950/agency/zing/zing-home.html (Accessed 12 January 2005)

Z39.50. (2003). Bib-1 Attribute Set, Z39.50: International Standards Maintenance Agency, Library of Congress, Washington DC. Available: http://www.loc.gov/z3950/agency/defns/bib1.html (Accessed 12 January 2005)

Z39.50. (2004). Z39.50: International Standards Maintenance Agency, Library of Congress, Washington DC. Available: http://www.loc.gov/z3950/agency/ (Accessed 12 January 2005) 
This paper is published in 'Program: electronic library and information systems', Vol.39, No.3, 2005, pp.227-247. 